\title{
Area-based socioeconomic factors and Human Papillomavirus (HPV) vaccination among teen boys in the United States
}

Kevin A. Henry ${ }^{1,2^{*}}$ (D), Allison L. Swiecki-Sikora ${ }^{3}$, Antoinette M. Stroup ${ }^{4,5}$, Echo L. Warner ${ }^{6}$ and Deanna Kepka $a^{6,7}$

\begin{abstract}
Background: This study is the first to examine associations between several area-based socioeconomic factors and human papillomavirus (HPV) vaccine uptake among boys in the United States (U.S.).

Methods: Data from the 2012-2013 National Immunization Survey-Teen restricted-use data were analyzed to examine associations of HPV vaccination initiation (receipt of $\geq 1$ dose) and series completion (receipt of three doses) among boys aged 13-17 years $(N=19,518)$ with several individual-level and ZIP Code Tabulation Area (ZCTA) census measures. Multivariable logistic regression was used to estimate the odds of HPV vaccination initiation and series completion separately.
\end{abstract}

Results: In 2012-2013 approximately 27.9\% (95\% Cl 26.6\%-29.2\%) of boys initiated and 10.38\% (95\% Cl 9.48\%-11.29\%) completed the HPV vaccine series. Area-based poverty was not statistically significantly associated with HPV vaccination initiation. It was, however, associated with series completion, with boys living in high-poverty areas ( $\geq 20 \%$ of residents living below poverty) having higher odds of completing the series (AOR 1.22, 95\% Cl 1.01-1.48) than boys in lowpoverty areas (0-4.99\%). Interactions between race/ethnicity and ZIP code-level poverty indicated that Hispanic boys living in high-poverty areas had a statistically significantly higher odds of HPV vaccine initiation (AOR 1.43, 95\% Cl 1.031.97) and series completion (AOR 1.56, 95\% Cl 1.05-2.32) than Hispanic boys in low-poverty areas. Non-Hispanic Black boys in high poverty areas had higher odds of initiation (AOR 2.23,95\% Cl 1.33-3.75) and completion (AOR 2.61,95\% Cl 1.06-6.44) than non-Hispanic Black boys in low-poverty areas. Rural/urban residence and population density were also significant factors, with boys from urban or densely populated areas having higher odds of initiation and completion compared to boys living in non-urban, less densely populated areas.

Conclusion: Higher HPV vaccination coverage in urban areas and among racial/ethnic minorities in areas with high poverty may be attributable to factors such as vaccine acceptance, health-care practices, and their access to HPV vaccines through the Vaccines for Children Program, which provides free vaccines to uninsured and under-insured children. Given the low HPV vaccination rates among boys in the U.S., these results provide important evidence to inform public health interventions to increase HPV vaccination.

Keywords: Human papillomavirus, HPV vaccination, Geographic factors, Cervical cancer, Cancer prevention, Health disparities

\footnotetext{
* Correspondence: khenry1@temple.edu

${ }^{1}$ Department of Geography, Temple University, 115 W. Polett Walk, 308

Gladfelter Hall, Philadelphia, PA 19122, USA

${ }^{2}$ Fox Chase Cancer Center, Cancer Prevention and Control Program, 333

Cottman Avenue, Philadelphia, PA 19111, USA

Full list of author information is available at the end of the article
}

(c) The Author(s). 2017 Open Access This article is distributed under the terms of the Creative Commons Attribution 4.0 International License (http://creativecommons.org/licenses/by/4.0/), which permits unrestricted use, distribution, and reproduction in any medium, provided you give appropriate credit to the original author(s) and the source, provide a link to the Creative Commons license, and indicate if changes were made. The Creative Commons Public Domain Dedication waiver (http://creativecommons.org/publicdomain/zero/1.0/) applies to the data made available in this article, unless otherwise stated. 


\section{Background}

In the United States (U.S.), approximately 39,000 HPVassociated cancers among women and men are diagnosed each year, many of which are preventable with the current HPV vaccine. Fifty-nine percent of these cancers occur in women [1]. HPV is the most common sexually transmitted infection in the U.S. [2] and is a known risk factor for genital warts and anal and oropharyngeal cancers in both men and women, cervical cancer in women, and penile cancer in men [1]. Over the past several years rates of $\mathrm{HPV}$-associated cancers among men have been increasing more rapidly than rates of HPV-associated cancers among women $[1,3,4]$.

Racial/ethnic and socioeconomic disparities in HPVassociated cancers have been documented in the U.S. Recent data indicate Hispanic men have higher rates of penile cancer compared to non-Hispanic $(\mathrm{NH})$ men. White and Black men have higher rates of oropharyngeal and anal cancers compared to men of other races $[1,5]$. Furthermore, incidence rates of HPV-associated cancers overall are highest among men living in census tracts with high poverty levels $(>20 \%)(\geq 20 \%$ of residents living below poverty) compared to men living in census tracts with low poverty levels ( $<5 \%$ of residents living below poverty) [6].

In 2006, the Centers for Disease Control and Prevention (CDC) Advisory Committee on Immunization Practices (ACIP) recommended that females aged 11 and 12 years receive a routine three-dose HPV vaccine [7]. ACIP expanded its recommendation of HPV vaccination in 2011 to include boys [8]. Vaccination is also recommended for females aged 13 through 26 years and males aged 13 through 21 years who were not vaccinated when they were younger [8]. Receipt of the vaccine at ages 11 and 12 builds an immune response to HPV before the average onset of sexual activity [9]. Prior to October 2016, HPV vaccines were recommended to be administered in three doses, through three intra-muscular injections, over a 6-month period [10], but ACIP now recommends that 11- to 12-year-olds receive two doses of HPV vaccine over a 6-month period [11].

Despite a safe and effective vaccine to reduce the risk of HPV-related cancers, HPV vaccination coverage in the U.S. remain low. The Healthy People's 2020 goal for teens completing the recommended HPV vaccine threedose series is $80 \%$ [12], yet in 2013 , only $57.3 \%$ of teen girls and $34.6 \%$ of teen boys had received at least one dose of HPV vaccine. Recent data also indicate that the HPV vaccination rates for teens vary by race/ethnicity and poverty level. Rates of HPV vaccine initiation and completion of the three-dose series are lower among non-Hispanics compared to Hispanics and other racial minorities and among teens whose families are living below the poverty line compared to their counterparts living above the poverty line [13].
The low U.S. HPV vaccination rates have led to numerous studies on HPV vaccination among teens to better understand the reasons for the low coverage. These studies suggest that the low number of HPV-immunized persons are in part due to lack of parental knowledge about HPV and the HPV vaccine, lack of health-care provider recommendations for the vaccine, missed opportunities for vaccination, religious and cultural factors, and beliefs that vaccinating adolescents against a sexually transmitted infection early is unnecessary and/or may promote sexual activity $[14,15]$.

The majority of research examining factors associated with HPV vaccination in the U.S. has focused on teen girls $[1,14,15]$. Furthermore, no studies have investigated the potential influence of geographic or area-based factors, such as the racial/ethnic composition, poverty, or population density, on HPV vaccination among boys. In other areas of cancer prevention, area-based factors, such as racial/ethnic composition, segregation, or areabased socioeconomic status (SES), have been shown to influence health status (e.g., cancer stage at diagnosis) $[16,17]$ and health behaviors (e.g., up-to-date on mammogram) independent of access to health insurance or an individual's income [18-20]. Similarly, a variety of area-based influences, including social context (e.g., social networks, similar norms of behavior, knowledge and perception of risk), physical circumstances (e.g., geographic accessibly), and economic conditions (e.g., access to health insurance and targeted interventions, time demands), could affect HPV vaccination uptake. For example, HPV vaccination uptake could be higher among teens living in lower-income ethnic minority communities due to resource availability from safety-net services, which provide free or subsidized vaccines, or from longterm targeted interventions [21, 22]. It is also possible that higher vaccination coverage could be a result of living among co-ethnics in segregated areas with similar cultural norms that promote vaccination. Conversely, economic burdens and barriers to health care among those living in poor areas could result in lower HPV vaccination initiation because of limited access to health resources and barriers to receiving preventive services [23-25]. Language barriers and lack of awareness about the benefits of the vaccine in ethnic minority communities [26-28] could also result in lower screening rates [29].

Given that uptake of cancer prevention and screening activities are generally higher among high-income, more educated populations [30-34], conventional wisdom suggests that uptake for a recommended vaccine that protects against some cancers would also follow this trend. However, a recent study noted that HPV vaccination uptake was highest for teen girls living in communities with high rates of poverty and among those with parents with low levels of education, and lowest for teen 
girls living in communities with low rates of poverty [35]. This was especially the case for Hispanic girls, who had a higher prevalence of HPV vaccination initiation than NH Black (NHB) and NH White (NHW) girls did. Given that the HPV vaccine was only recently recommended for boys in 2011, it is unknown whether areabased factors are associated with HPV vaccination uptake among boys as they are for girls.

In this study, we analyzed data from the National Immunization Survey-Teen (NIS-Teen) to examine associations between both individual-level and area-based factors and HPV vaccine initiation and completion among boys. This study is the first to explore area-based factors that may be associated with HPV vaccine uptake among boys in the U.S.

\section{Methods}

\section{Study design}

We conducted a secondary data analysis of restricteduse data from the 2012 and 2013 NIS-Teen, an annual survey conducted by the CDC to monitor vaccination uptake in the U.S. The NIS-Teen includes a nationally representative stratified sample of girls and boys aged 13 to 17 years in all 50 states and the District of Columbia. The survey is based on random-digit dialing of both landline and cellular telephone numbers to identify eligible households. It includes two parts: 1) a survey of parents or guardians of 13- to 17-year-olds to collect information about demographic and socioeconomic characteristics and 2) a survey mailed to all vaccination providers whom the parents identified and consented to verification of their teen's immunization histories. The survey sampling procedures have been described elsewhere [36]. The National Center of Health Statistics (NCHS) Research Ethics Review Board (ERB) approved data collection for NIS-Teen. Analysis of de-identified data from the survey is exempt from the federal regulations for the protection of human research participants. The NCHS ERB also approved analysis of restricted data which was completed at a NCHS Research Data Center. The 2012-2013 NIS-Teen included 34,931 boys aged 13 to 17 years with completed surveys in the U.S. (excluding U.S. Virgin Islands). Of the 34,931 boys, 20,355 (55.9\%) had adequate provider-verified vaccination records and the 14,576 had only parental reported vaccination status which is subject to recall error [36]. Adequate provider verified vaccination data means that sufficient vaccination history information was obtained from the provider(s) to determine whether the teen is up-to-date with respect to the recommended vaccination schedule. Because of children with adequate provider data have certain factors believed to be associated with a greater likelihood of being up-to-date, compared with children who had missing provider data the developers of the NIS-Teen survey have reduced potential bias in the provider verified records by applying a weighting adjustment to the provider verified records [36]. Detailed information about the NIS-Teen survey and details about adjustments for bias can be found in the NIS-Teen users guide [36].

The present study was limited to only providerverified vaccination records. An additional 3.3\% of these cases were excluded from the statistical models because of missing ZIP codes (i.e., postal codes) and $0.9 \%$ were excluded. There were no differences between participants in the final sample and those excluded with regard to any of the individual-level or area-based socioeconomic measures. The final dataset included 19,518 boys.

\section{Measures}

\section{Individual-level variables}

We examined two primary HPV vaccination outcomes that were based on provider-verified vaccination records: 1) initiation: receipt of at least one dose of the threedose HPV vaccine series; and 2) completion: receipt of three doses of HPV vaccine. We included several individual-level variables previously shown to be associated with HPV vaccine uptake [14, 37-41]. The following NIS-Teen variables based on survey questions completed by the parents were included: (a) the teen's current age in years; (b) race/ethnicity (NHW, NHB, $\mathrm{NH}$ Other, and Hispanic); (c) health insurance type (employer or union, Medicaid, or the State Children's Health Insurance Program [SCHIP]; military or Indian Health Service [IHS], and no insurance); (d) poverty status (categorized as above poverty, high income [annual income $>\$ 75,000$ ]; above poverty, moderate income [annual income $\leq \$ 75,000]$; below poverty, based on the U.S. Census family poverty thresholds [42]; and unknown); (e) receipt of a provider recommendation for HPV vaccination (yes, no, or don't know); (f) mother's age ( $\leq 34$ years, $35-44$, or $\geq 45$ ); (g)mother's marital status (currently married or not currently married); and (h) mother's education lev( $<12$ years; 12 years with high school diploma or general equivalency diploma; $>12$ years without college degree; or college degree or higher). We also included one factor from the health-care system level based on the provider survey: facility type (all private facilities, all public facilities, all hospital facilities, all STD/school/teen clinics, or other facilities, plus mixed and unknown) where vaccines were administered.

\section{Area-based variables}

Several area-based socioeconomic measures created using U.S. Census ZIP Code Tabulation Areas (ZCTAs) data were merged with the ZIP codes of participants' current residences at the CDC Research Data Center (RDC). ZIP codes are restricted variables; therefore, 
these data were accessed through the RDC. The ZCTAs are generalized areal representations of U.S. Postal Service areas, and in most instances the ZCTA is the same as the ZIP code [43]. ZCTAs were used for this study because smaller geographic areas, such as census tracts, are not available in the NIS-Teen restricted dataset.

We included several area-based measures previously incorporated in health disparities research $[44,45]$ and research on vaccination uptake and use of cancer screening services [16, 46-48]. For this study, ZCTA poverty was conceptualized as an area- or communitybased socioeconomic measure. Area-based socioeconomic measures describe a geographically defined area (e.g., census tracts, ZCTAs) in which an individual lives and that could affect health and access to care through several pathways, including the material resources available (e.g., community health centers), social capital, and social networks (e.g., social contagion, similar norms of behavior) $(32,33,44)$. Area-based socioeconomic measures capture conditions that affect all individuals living in the same area and have been shown to be an independent predictor of health outcomes (26-28). SES was based on poverty status derived from the 2008-2012 U.S. Census American Community Survey (ACS). It was grouped into four categories according to the percentage of the population in the ZCTA living below the federally defined poverty threshold: less than $5 \%, 5-9.9 \%, 10$ $19.9 \%$, and $20 \%$ or greater, with the last category considered a severely disadvantaged area.

A measure of racial/ethnic composition or density of a ZCTA was also created. Racial/ethnic composition refers to proportions of people of the same race/ethnicity in a defined area and has been used as a proxy measure of segregation $[49,50]$. Segregation refers to the degree to which two or more racial/ethnic groups live separately from one another in a geographically defined area [50]. We used this measure because of our interest in knowing the majority racial/ethnic group in each ZCTA and because area-based socioeconomic measures also vary by racial/ethnic composition in the U. S, with higher socioeconomic areas generally having fewer Blacks and Hispanics and more Whites and Asian/Pacific Islanders (API). Numerous studies have shown that persistent residential racial/ethnic segregation of either poor Blacks or Hispanics in U.S. communities serves as both a health promoter by facilitating stronger social support networks and a health barrier by fostering conditions that limit financial, educational, or social resources [16, 45, 51, 52]. Racial composition was based on the U.S. Census ACS estimates of the percentage of each racial/ethnic group in each ZCTA. Using the percentages, we categorized the majority racial/ethnic group in each ZCTA: $>50 \%$ Hispanic, $>50 \% \mathrm{NHW},>50 \% \mathrm{NH}$ Asian/Pacific Islander (NHAPI), $>50 \% \mathrm{NHB},>50 \% \mathrm{NH}$ American Indian/
Native Alaskan, or NH Mixed if not any of the previous. We combined NH American Indian/Native Alaskan with $\mathrm{NH}$ Asian/Pacific Islander into a category called $\mathrm{NH}$ Other due to small numbers.

We also included a ZCTA measure of population density (total ZCTA population counts divided by land area), which has been used in previous research as an indicator of the built environment, crowding [53] and a proxy of urban/rural residence. ZCTA population density was divided into quartiles based on the nationwide geographic distribution (Q1 1-20, Q2 21-71, Q3 72-651, Q4 > 651 people per square mile) and merged with survey participants based on ZIP code. We also included ZCTA measures of rural and urban residence based on the rural-urban commuting area codes (RUCAs). The RUCAs provided a definition of rural/urban residences based on criteria that included population density and population work-commuting patterns. We categorized this variable as: (1) urban, (2) large rural city/town, (3) small rural town, and (4) isolated small rural town [54].

\section{Statistical analysis}

NIS-Teen data for years 2012 and 2013 were combined and the sampling weights for the provider verified data (PROVWT) was recalculated using suggested methods in the NIS-Teen Users Guide [36] to ensure the sampling weights were appropriately adjusted for calculating the weighted percentages and effect estimates.

We performed bivariate association tests between all the variables and our two primary HPV vaccination outcomes (initiation and completion) with Wald chi-square tests. We also used logistic regression to identify variables associated with our two primary HPV vaccination outcomes. For each outcome separately, we entered all variables associated with the outcome in bivariate models $(p<0.05)$ into a multivariable logistic regression model. The multivariate models produced adjusted odds ratios (AORs) and 95\% confidence intervals (CIs). One multivariable model included only individual and provider factors, and two separate multivariable models were run to examine the independent associations of ZCTA poverty and racial/ethnic composition with vaccination outcomes. In the two separate multivariable models that included area-based measures we also included population density and in an additional analysis replaced population density with rural/urban residence. Joint contributions of area poverty, racial composition, and race/ethnicity were assessed with interaction terms between individual-level race/ethnicity and ZCTA poverty and individual-level race/ethnicity and ZCTA racial composition. Population density was also included in the multivariable models that were run to examine the associations of ZCTA poverty and racial/ethnic composition with vaccination outcomes. The surveyed state was 
included as a random effect to account for dependency by state residence (e.g., state health programs that will influence the boys from that state).

Bivariate associations were analyzed using procedures for complex survey data in SAS statistical software 9.3 (i.e., PROC SURVEYFREQ) [55]. Logistic regression analyses were conducted using SAS GLIMMIX, which implements the generalized linear mixed model and allows for the incorporation of stratum-specific weighted analysis [56]. Statistical tests were two-tailed with a critical alpha of .05.

\section{Results}

\section{Survey participant characteristics}

Sociodemographic and geographic characteristics of the survey participants are presented in Table 1 . Overall, the age distribution among boys included in the survey was about even, with each age group making up $19 \%$ or $20 \%$ of the sample. Most boys were NHW (55.2\%), from urban areas $(89.0 \%)$ with population densities greater than 651 persons per square mile (quartile 4; 59.5\%), and in predominately NHW (70.6\%) ZCTAs. At the time of the survey, most mothers were $>35$ years old $(90.2 \%)$, married (65.5\%), did not have a college degree (64.6\%), and had employer- or union-provided health insurance (45.9\%). Only a third of the parents (35.0\%) received a recommendation from their health-care providers to have their sons vaccinated against HPV.

\section{Initiation}

Overall, for the years 2012-2013, 27.9\% of boys received at least one dose of HPV vaccine (Table 1). In the bivariate analysis, all the individual and geographic variables were significantly associated with HPV vaccine initiation except for the boy's age and facility types for providers (Table 1). Based on multivariable analysis that included only individual-level variables, boys with health insurance through Medicaid/SCHIP or IHS/military insurance had significantly higher odds of HPV vaccine initiation than those with employer- or union-provided insurance (Model 1, AOR 1.53, 95\% CI 1.36-1.71; AOR 1.38, 95\% CI 1.22-1.56, respectively) (Table 2). Among boys whose parents received a provider recommendation to vaccinate, the odds of HPV vaccination initiation were 9.4 times higher (95\% CI 8.66-10.20) than for boys without such a recommendation. Compared to boys whose mothers had a college degree, boys whose mothers had less than 12 years of education had higher odds of vaccine initiation (AOR 1.25, 95\% CI 1.09-1.44). However, boys whose mothers were high school graduates or completed some college had significantly lower odds of initiating vaccination (AOR 0.83, 95\% CI 0.74-0.93). Boys from households with incomes below the poverty threshold had higher odds of HPV vaccine initiation compared to households with incomes above the poverty threshold (AOR 1.35, 95\% CI 1.17-1.55). Hispanic, NHB, and NH Other had an adjusted odds of initiation of 2.14 (95\% CI 1.92-2.39), 1.72 (95\% CI 1.53-1.93), and 1.22 (95\% CI 1.06-1.40) times greater than NHW boys, respectively.

In the multivariable model for initiation (Table 2, Model 3), which included individual-level factors and ZCTA-level poverty and population density, the odds of initiation were highest among boys from the highest poverty category compared to the lowest category, but the result was not statistically significant. The highest population density category (Q4) compared to the lowest population density category (Q1) was not statistically significant; however the highest category (Q4) versus the second (Q2) and third category (Q3) were significant (Model 3, AOR 1.32, 95\% CI 1.14-1.52; 1.12 95\% 95\%CI $1.121 .02-1.23$, respectively). We also separately examined the geographic variable rural/urban residence by including it in Model 3 instead of population density (model results not shown in table). Boys from urban areas had higher odds of initiation when compared to those from isolated small rural towns (reference group) (AOR 1.38, 95\% CI 1.04-1.83), small rural towns (reference group) (AOR 1.38, 95\% CI 1.09-1.76), and large rural towns (reference group) (AOR 1.50, 95\% CI 1.251.80).

Figure 1 summarizes the model-adjusted percentage of boys who initiated the HPV vaccination series based on the interaction term race/ethnicity $\times$ ZCTA poverty included in the model, and Fig. 2 summarizes the odds ratios for the statistically significant interactions for the same model. Hispanic boys from the most impoverished ZCTAs ( $\geq 20 \%$ of residents below poverty) had higher odds of HPV vaccination initiation (AOR 1.43, 95\%CI 1.03-1.97) than Hispanic boys from the least impoverished ZCTAs $(0-4.9 \%$ of residents below poverty) (Fig. 2). NHB boys from the most impoverished ZCTAs had higher odds of initiation (AOR 2.23, 95\% CI 1.333.75) than NHB boys from the least impoverished ZCATAs. Hispanic boys from the most impoverished ZCTAs also had higher odds of initiation than did NHB (AOR 1.32, 95\% CI 1.09-1.59) and NHW (AOR 2.84, 95\% CI 2.32-3.46) boys also from the most impoverished ZCTAs. Conversely, NHWs from the most impoverished ZCTAs had lower odds than NHWs from the least impoverished ZCTAs to initiate (AOR 0.80, 95\% CI 0.650.97). The interaction term individual-level race/ethnicity $\times$ racial composition was not statistically significant.

\section{Completion}

Overall, for the years 2012-2013, 10.4\% of boys completed the recommended HPV vaccine regimen (Table 1). In the bivariate analysis, all the individual and 
Table 1 Individual-level and area-based characteristics of HPV Vaccine Initiation (Receipt of at Least One Dose) and completion (receipt of $\geq 3$ doses): Teen Boys Aged 13 to 17 based on responses from the National Immunization Survey-Teen, 2012-2013

\begin{tabular}{llll}
\hline Characteristics & $\begin{array}{l}\text { Survey Participants, } \\
\mathrm{n} \text { weighted } \%\end{array}$ & $\begin{array}{l}\text { Weighted \% }(95 \% \mathrm{Cl}), \\
\text { Vaccine Initiation }(\geq 1 \text { dose })\end{array}$ & $\begin{array}{l}P \text {-value } \\
\text { Weighted \% }(95 \% \mathrm{Cl}), \text { Vaccine } \\
\text { Completion }(\geq 3 \text { doses })\end{array}$ \\
\hline Total & 19,518 & $27.9(26.6-29.2)$ & $10.38(9.48-11.29)$ \\
Year & & & $<.0001$ \\
2012 & 10,265 & $20.8(19.24-22.35)$ & $6.79(5.84-7.74$ \\
2013 & 9253 & $35.26(33.23-37.29)$ & $13.64(12.24-15.05)$
\end{tabular}

Individual-level variables

Age

13

14

15

16

17

Mother's marital status

Married

Not married

Mother's age, years

$<=34$

35 TO 44

$>=45$

Type of insurance coverage

No insurance

Employer or union

SCHIP or Medicaid

IHS, military, other

Mother's education, years

$<12$ years

12 years

$>12$ years, non-college graduate

College graduate

Poverty Status

Above poverty, Annual Income $>\$ 75,000$

Above poverty, Annual Income $\leq \$ 75,000$

Below poverty

Unknown poverty status

Race/ethnicity of teen

Hispanic

Non-Hispanic White

Non-Hispanic Black

Non-Hispanic other + multiple race

\section{$3942(19.99)$ \\ 4117 (20.54) \\ $3884(20.63)$ \\ $3911(19.66)$ \\ 3664 (19.18)}

$14,314(65.46)$

$5204(34.54)$

$1590(9.77)$

$8112(46.59)$

$9816(43.64)$

1269 (8.43)

$10,645(45.86)$

4957 (33.39)

2647 (12.32)

$2012(13.93)$

3615 (24.97)

$5451(25.69)$

$8440(35.41)$

8574 (33.48)

7165 (38.07)

$3225(24.16)$

$554(4.29)$

2748 (22.18)

$13,050(55.23)$

1860 (13.93)

1860 (8.66)

Received provider recommendation to get HPV vaccine

Yes

No

Don't Know
$7121(35.01)$

$10,793(57.49)$

1399 (7.50)
0.7461

0.2899

$26.4(23.58-29.23)$

$28.26(25.35-31.18)$

$28.09(25.38-30.80)$

$28.50(25.79-31.20)$

$26.58(23.77-29.39)$

25.39 (23.94-26.84)

$31.72(29.40-34.05)$

$34.55(30.46-38.65)$

$28.09(26.11-30.07)$

$25.47(23.78-27.16)$

25.9 (21.22-30.59)

21.45 (20.03-22.87)

35.91 (33.34-38.48)

$28.96(25.43-32.48)$

40.97 (35.79-44.15)

27.94 (25.08-30.79)

$22.26(20.14-24.39)$

25.92 (24.20-27.63)

$22.86(21.31-24.42)$

24.85 (22.82-26.88)

37.93 (34.92-40.95)

$30.29(22.48-38.10)$

40.75 (37.11-44.40)

$20.67(19.50-21.85)$

34.26 (30.58-37.93)

$27.13(23.55-30.71)$

$53.66(51.48-55.83)$

12.21 (10.90-13.52)

$25.18(21.11-29.24)$
$9.11(7.48-10.74)$

$9.38(7.47-11.29)$

$11.52(9.38-13.67)$

$9.66(7.85-11.46)$

$11.18(9.21-13.15)$

$<.001$

$<.3775$

$9.88(8.85-10.91)$

$10.71(9.20-12.21)$

$<.0002$

$11.58(8.93-14.23)$

$9.36(8.11-10.61)$

$10.71(9.42-12.01)$

$<.0001$

$5.73(3.91-7.54)$

8.34 (7.31-9.37)

$13.39(11.56-15.22)$

$11.29(8.72-13.86)$

$<.0001$

$17.02(13.63-20.41)$

$9.09(7.29-10.90)$

$8.29(6.79-9.78))$

9.59 (8.54-10.65)

$<.0001$

8.93 (7.89-9.98)

$9.35(7.91-10.79)$

$13.10(11.12-15.08)$

10.56 (4.29-16.83)

$<.0001$

$<.0001$

16.14 (13.30-18.97)

7.73 (6.95-8.51)

10.46 (8.29-12.63)

$9.95(7.79-12.10)$

$<.0001$

$<.0001$
21.09 (19.20-22.98)

3.86 (3.05-4.67)

$8.68(6.07-11.29)$ 
Table 1 Individual-level and area-based characteristics of HPV Vaccine Initiation (Receipt of at Least One Dose) and completion (receipt of $\geq 3$ doses): Teen Boys Aged 13 to 17 based on responses from the National Immunization Survey-Teen, 2012 -2013 (Continued)

\begin{tabular}{|c|c|c|c|c|}
\hline \multicolumn{5}{|l|}{ Healthcare system factor } \\
\hline \multicolumn{2}{|l|}{ Facility types for teen's providers } & & $<.8268$ & $<.8739$ \\
\hline All public facilities & $2722(14.44)$ & $26.58(22.86-30.31)$ & $9.26(6.64-11.87)$ & \\
\hline All hospital facilities & $1884(8.18)$ & $28.97(24.67-33.27)$ & $10.23(7.79-12.67)$ & \\
\hline All private facilities & $9086(50.77)$ & $27.26(25.57-28.96)$ & $10.44(9.25-11.62)$ & \\
\hline $\begin{array}{l}\text { Mixed \& STD/school/teen clinics or other } \\
\text { facilities }\end{array}$ & $5250(23.53)$ & $28.64(26.17-31.12)$ & $9.88(8.44-11.32)$ & \\
\hline Unknown & $496(3.08)$ & $28.28(19.44-37.12)$ & $13.01(4.49-21.53)$ & \\
\hline \multicolumn{5}{|l|}{ ZCTA area-based measures } \\
\hline \multicolumn{2}{|c|}{ Racial Composition (50\% + of specific race/ethnic group) } & & $<.0001$ & $<.0002$ \\
\hline Hispanic & $1150(10.24)$ & $47.13(41.30-52.97)$ & $16.58(11.93-21.22)$ & \\
\hline Mixed & $1783(12.34)$ & $33.02(28.80-37.24)$ & $13.66(10.84-16.48)$ & \\
\hline Non-Hispanic Black & $1083(5.95)$ & $33.27(28.83-37.71)$ & $9.88(7.06-12.71)$ & \\
\hline Non-Hispanic White & $15,155(70.61)$ & $23.23(22.03-24.43)$ & $8.69(7.86-9.51)$ & \\
\hline Non-Hispanic other & $245(0.85)$ & $36.12(15.79-56.46)$ & $6.26(2.35-10.16)$ & \\
\hline \multicolumn{2}{|l|}{ Poverty (\% below poverty) } & & $<.0001$ & $<.0217$ \\
\hline $0-4.99 \%$ low & $2981(13.47)$ & $24.36(21.58-27.13)$ & $9.01(7.01-11.02)$ & \\
\hline $5-9.9 \%$ & $5192(25.16)$ & $25.54(23.30-27.78)$ & $9.52(8.07-10.98)$ & \\
\hline $10-19.9 \%$ & $7170(36.44)$ & $25.32(23.20-27.44)$ & $9.14(7.82-10.46)$ & \\
\hline $20+\%$, high & $4070(24.93)$ & $34.76(31.96-37.57)$ & $12.92(10.78-5.06)$ & \\
\hline \multicolumn{2}{|c|}{ Population density quartiles (people per square mile) } & & $<.0001$ & $<.0001$ \\
\hline Q1 1-20 (lowest density) & $1472(3.25)$ & $19.32(15.53-23.12)$ & $8.10(5.08-11.12)$ & \\
\hline Q2 21-71 & $2516(10.29)$ & $20.41(17.11-23.72)$ & $5.63(3.99-7.28)$ & \\
\hline Q3 72-651 & $5504(26.97)$ & $23.44(21.24-25.65)$ & $9.44(7.79-11.09)$ & \\
\hline Q4 > 651 (highest density) & $9924(59.49)$ & 31.17 (29.44-32.91) & $11.38(10.21-12.56)$ & \\
\hline \multicolumn{2}{|l|}{ Residence type } & & $<.0001$ & $<.0001$ \\
\hline Isolated Small Rural Town & $964(2.32)$ & $17.34(13.51-21.16)$ & $5.31(3.33-7.30)$ & \\
\hline Small Rural Town & $938(3.07)$ & $18.70(13.94-23.45)$ & $5.50(3.32-7.67)$ & \\
\hline Large Rural Town & $1715(5.66)$ & $18.55(15.63-21.47)$ & $6.45(4.69-8.21)$ & \\
\hline Urban focused & 15,799 (88.95) & $28.74(27.38-30.11)$ & $10.68(9.74-11.63)$ & \\
\hline
\end{tabular}

Note. Cl confidence interval, HPV human papillomavirus, IHS Indian Health, SCHIP State Children's Health Insurance Program; Frequencies (n) were not weighted; Percent's weighted based on sampling weight

${ }^{\text {a }}$ Poverty status was based on the US Census poverty thresholds for 2012 and 2013

geographic variables were statistically significantly associated with HPV vaccine completion except boy's age, mother's marital status, mother's age, and facility type for providers. In the multivariable analysis including only individual-level variables (Table 3, Model 1), boys with SCHIP/Medicaid (AOR 1.37, 95\% CI 1.18-1.59) or IHS/ military health insurance (AOR 1.27, 95\% CI 1.08-1.49) had higher odds of HPV vaccine completion than those with employer- or union-provided insurance. For boys whose parents received a provider recommendation to vaccinate, the odds of vaccination completion were 6.53 times higher (95\% CI 5.83-7.33) than for boys without the recommendation. Higher odds of vaccination completion were also associated with boys whose mothers had $<12$ years of education compared to those with a college degree (AOR 1.73, 95\% CI 1.45-2.07). Hispanic, $\mathrm{NHB}$, and $\mathrm{NH}$ Other boys had completion rates 1.95 (AOR 95\% CI 1.70-2.24), 1.26 (AOR 95\% CI 1.08-1.48), and 1.22 (AOR 95\% CI 1.01-1.47) times greater than NHW boys, respectively. There were no significant associations between poverty level and HPV vaccination completion.

In the multivariable model for completion (Table 2, Model 3), which included individual-level factors and ZCTA poverty and population density, the odds of completion were statistically significantly higher among boys from the highest poverty category compared to boys from the lowest poverty category (Table 3, Model 3, 
Table 2 Odds of HPV Vaccine initiation (Receipt of at Least One Dose) among of Teen Boys 13 to 17 Years of age for Individual-level individual-level and area-based measures: National Immunization Survey-Teen, 2012-2013

\begin{tabular}{|c|c|c|c|c|}
\hline \multirow[b]{2}{*}{ Characteristics } & \multicolumn{2}{|c|}{ HPV vaccine initiation ${ }^{a}$} & \multirow[b]{2}{*}{$\begin{array}{l}\text { Individual-level + ZCTA } \\
\text { racial composition + ZCTA } \\
\text { population density (Model 2), } \\
\text { AOR }^{b, c}(95 \% \mathrm{Cl})\end{array}$} & \multirow[b]{2}{*}{ 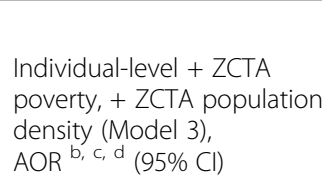 } \\
\hline & $\begin{array}{l}\text { Undadjusted }^{\mathrm{b}} \\
\text { OR }(95 \% \mathrm{Cl})\end{array}$ & $\begin{array}{l}\text { Individual-level only, } \\
\text { (Model 1) AOR b,c } \\
(95 \% \mathrm{Cl})\end{array}$ & & \\
\hline \multicolumn{5}{|l|}{ Individual-level variables } \\
\hline \multicolumn{5}{|l|}{ Age } \\
\hline 13 & Ref & Ref & Ref & Ref \\
\hline 14 & $1.14(1.03,1.26)^{*}$ & $1.30(1.16,1.46)^{* *}$ & $1.30(1.15,1.46)^{* *}$ & $1.30(1.15,1.46)^{* *}$ \\
\hline 15 & $1.12(1,01,1.23)^{*}$ & $1.36(1.21,1.53)^{* *}$ & $1.34(1.19,1.51)^{* *}$ & $1.35(1.20,1.51)^{* *}$ \\
\hline 16 & $1.18(1.07,1.31)^{*}$ & $1.40(1.24,1.58)^{* *}$ & $1.40(1.24,1.58)^{* *}$ & $1.40(1.24,1.58)^{* *}$ \\
\hline 17 & $1.03(0.93,1.15)$ & $1.32(1.17,1.49)^{* *}$ & $1.31(1.16,1.48)^{* *}$ & $1.31(1.16,1.48)^{* *}$ \\
\hline \multicolumn{5}{|l|}{ Mother's age, years } \\
\hline$<=34$ & $1.17(1.10,1.26)^{* *}$ & $1.12(1.03,1.22)^{* *}$ & $1.13(1.04,1.23)^{* *}$ & $1.13(1.04,1.23)^{* *}$ \\
\hline 35 TO 44 & $1.63(1.46,1.82)^{* *}$ & $1.33(1.16,1.52)^{*}$ & $1.33(1.16,1.53)^{*}$ & $1.33(1.16,1.53)^{*}$ \\
\hline$>=45$ & Ref & Ref & Ref & Ref \\
\hline \multicolumn{5}{|l|}{ Type of insurance coverage insurance } \\
\hline No insurance & $1.26(1.11,1.42)^{*}$ & $1.17(0.97,1.43)$ & $1.15(0.94,1.40)$ & $1.16(0.99,1.36)$ \\
\hline Employer or union & Ref & Ref & Ref & Ref \\
\hline SCHIP or Medicaid & $2.04(1.89,2.20)^{* *}$ & $1.53(1.36,1.71)^{* *}$ & $1.53(1.37,1.71)^{* *}$ & $1.54(1.38,1.73)^{* *}$ \\
\hline IHS, military, other & $1.43(1.29,1.58)^{* *}$ & $1.38(1.22,1.56)^{* *}$ & $1.37(1.21,1.55)^{* *}$ & $1.38(1.22,1.56)^{* *}$ \\
\hline \multicolumn{5}{|l|}{ Mother's education, years } \\
\hline$<12$ years & $1.90(1.73,2.09)^{* *}$ & $1.25(1.09,1.44)^{*}$ & $1.23(1.07,1.42)^{*}$ & $1.25(1.09,1.44)^{*}$ \\
\hline 12 years & $1.13(1.04,1.22)^{*}$ & $0.83(0.74,0.93)^{*}$ & $0.84(0.75,0.94)^{*}$ & $0.84(0.75,0.94)^{*}$ \\
\hline$>12$ years, non-college graduate & $0.84(0.77,0.92)^{* *}$ & $0.66(0.60,0.74)^{* *}$ & $0.67(0.60,0.74)^{* *}$ & $0.67(0.60,0.74)^{* *}$ \\
\hline College graduate & Ref & Ref & Ref & Ref \\
\hline \multicolumn{5}{|l|}{ Poverty status } \\
\hline Above poverty, $>\$ 75,000$ & Ref & Ref & Ref & Ref \\
\hline Above poverty, $\leq \$ 75,000$ & $1.17(1.08,1.27)^{* *}$ & $0.99(0.89,1.10)$ & $1.00(0.90,1.12)$ & $0.10(0.90,1.11)$ \\
\hline Below poverty & $2.13(1.96,2.32)^{* *}$ & $1.35(1.17,1.55)^{* *}$ & $1.34(1.16,1.54)^{* *}$ & $1.34(1.17,1.55)^{* *}$ \\
\hline Unknown poverty status & $1.50(1.27,1.76)^{* *}$ & $1.18(0.97,1.43)$ & $1.15(0.94,1.40)$ & $1.18(0.97,1.44)$ \\
\hline \multicolumn{5}{|l|}{ Race/ethnicity of teen } \\
\hline Hispanic & $2.42(2.22,2.63)^{* *}$ & $2.14(1.92,2.39)^{* *}$ & $1.88(1.68,2.12)^{* *}$ & $2.02(1.81,2.26)^{* *}$ \\
\hline Non-Hispanic White & Ref & Ref & Ref & Ref \\
\hline Non-Hispanic Black & $2.07(1.88,2.28)^{* *}$ & $1.72(1.53,1.93)^{* *}$ & $1.57(1.38,1.78)^{* *}$ & $1.60(1.41,1.80)^{* *}$ \\
\hline Non-Hispanic other and multiple race & $1.31(1.16,1.48)^{* *}$ & $1.22(1.06,1.40)^{*}$ & $1.19(1.03,1.37)^{*}$ & $1.18(1.02,1.36)^{*}$ \\
\hline \multicolumn{5}{|c|}{ Received provider recommendation to get HPV vaccine } \\
\hline Yes & $8.38(7.77,9.03)^{* *}$ & $9.40(8.66,10.20)^{* *}$ & $9.34(8.61,10.15)^{* *}$ & $9.34(8.60-10.14)^{* *}$ \\
\hline No & Ref & Ref & Ref & Ref \\
\hline Don't know & $2.41(2.36,2.47)^{* *}$ & $2.40(2.09,2.76)^{* *}$ & $2.39(2.08,2.75)^{* *}$ & $2.39(2.07-2.75)^{* *}$ \\
\hline \multicolumn{5}{|l|}{ ZCTA Area-based measures } \\
\hline \multicolumn{5}{|c|}{ Racial composition ( $50 \%$ + of that groupin ZCTA) } \\
\hline Hispanic & $2.62(2.35,2.93)$ & - & $1.41(1.22,1.63)^{* *}$ & - \\
\hline Mixed & $1.47(1.32,1.62)$ & - & $1.00(0.88,1.13)$ & - \\
\hline Non-Hispanic Black & $1.76(1.54,2.02)$ & - & $1.15(0.97,1.36)$ & - \\
\hline
\end{tabular}


Table 2 Odds of HPV Vaccine initiation (Receipt of at Least One Dose) among of Teen Boys 13 to 17 Years of age for Individual-level individual-level and area-based measures: National Immunization Survey-Teen, 2012-2013 (Continued)

\begin{tabular}{|c|c|c|c|c|}
\hline Non-Hispanic White & Ref & - & Ref & Ref \\
\hline Non-Hispanic other & $1.44(1.03,2.01)$ & - & $0.96(0.63,1.44)$ & - \\
\hline \multicolumn{5}{|l|}{ ZCTA poverty (\% below poverty) C } \\
\hline 0-4.99\%, least impoverished & Ref & - & - & Ref \\
\hline $5-9.9 \%$ & $1.08(0.97,1.21)$ & - & - & $1.04(0.92,1.19)$ \\
\hline $10-19.9 \%$ & $1.15(1.03,1.29)^{*}$ & - & - & $0.95(0.83,1.08)$ \\
\hline $20+\%$, poorest & $1.77(1.58,1.98)^{* *}$ & - & - & $1.13(0.98,1.31)$ \\
\hline \multicolumn{5}{|c|}{ ZCTA Population density quartiles (people per square mile) } \\
\hline Q1 1-20 (lowest density) & Ref & - & Ref & Ref \\
\hline Q2 21-71 & $1.12(0.88,1.41)$ & - & $0.95(0.74,1.24)$ & $0.97(0.75-1.25)$ \\
\hline Q3 72-651 & $1.26(1.02,1.56)^{*}$ & - & $1.12(0.88,1.43)$ & $1.14(0.89-1.45)$ \\
\hline Q4 > 651 (highest density) & $1.70(1.37,2.10)^{* *}$ & & $1.26(0.99,1.60)$ & $1.27(1.00-1.62)$ \\
\hline Q4 vs Q2 & $1.52(1.35,1.72)^{* *}$ & & $1.33(1.04-1.25)^{*}$ & $1.32(1.14-1.52)^{*}$ \\
\hline Q4 vs Q3 & $1.35(1.25,1.46)^{*}$ & & $1.14(1.25-1.04)^{*}$ & $1.12(1.02-1.23)^{*}$ \\
\hline \multicolumn{5}{|l|}{ ZCTA -Residence type } \\
\hline 1. Isolated Small Rural T & Ref & & - & - \\
\hline 2. Small Rural Town & $1.13(0.82,1.57)$ & & - & - \\
\hline 3. Large Rural Town & $1.10(0.82,1.48)$ & & - & - \\
\hline 4. Urban focused & $1.71(1.33,2.21)^{* *}$ & & - & - \\
\hline
\end{tabular}

Note $\mathrm{Cl}$ confidence interval, HPV human papillomavirus, IHS Indian Health, SCHIP State Children's Health Insurance Program, Ref Reference, AOR adjusted odds ratio

${ }^{*} P<.05 ;{ }^{*} P<.0001$

a initiation $=>=1$ dose

b Unadjusted odds and models 1-3 were weighted based on sampling weight and also included state random effects. The sample size was $n=19,188$

'Multivariable models 1-3 include all of the variables without dashes ( - ) and also include the variables, marital status, mother's age, survey year, and teen's current age in years and state random effects

${ }^{d}$ Poverty status was based on the US Census poverty thresholds for 2012 and 2013

AOR 1.22, 95\% CI 1.01-1.48). The highest population density category (Q4) compared to those from the lowest population density category (Q1) was not statistically significant; however the highest category (Q4) versus the second (Q2) was significant (Model 3, AOR 1.57, 95\% CI 1.27-1.94). We also examined the geographic variable rural/urban residence by including it in Model 3 instead of population density (model results not shown in table). Boys from urban areas had higher odds of completion when compared to those from isolated small rural towns (reference group) (AOR 1.46, 95\% CI 0.96-2.24), small rural towns (reference group) (AOR 1.50, 95\% CI 1.052.18 ), and large rural towns (reference group) (AOR 1.40, 95\% CI 1.08-1.81).

Figure 1 summarizes the model-adjusted percentage of boys who completed the HPV vaccination series based on the interaction term race/ethnicity $\times$ ZCTA poverty included in the model, and Fig. 2 summarizes the odds ratios for the statistically significant interactions for the same model. Hispanic boys from the most impoverished ZCTAs ( $\geq 20 \%$ of residents below poverty) had higher odds of HPV vaccination completion (AOR 1.56, 95\% CI 1.05-2.32) than did Hispanic boys from the least impoverished ZCTAs $(0-4.9 \%$ of residents below poverty) (Fig. 2). NHB boys from the most impoverished ZCTAs had higher odds of completion (AOR 2.61, 95\% CI 1.06-6.44) than NHB boys from the least impoverished ZCTAs codes had. Hispanic boys from the most impoverished ZCTAs also had higher odds of completion than did NHB (AOR 1.91, 95\% CI 1.50-2.43) and NHW (AOR 2.68, 95\% CI 2.05-3.49) boys also from the most impoverished ZCTAs. Conversely, NHWs from the most impoverished ZCTAs had lower odds of completion than NHWs from the least impoverished ZCTAs did, although not significant (AOR 0.83, 95\% CI 0.631.10) (Fig. 2). The interaction term individual-level race/ ethnicity $\times$ racial composition was not statistically significant.

\section{Discussion}

HPV vaccination is low among teen boys in the U.S. despite increasing numbers of HPV-related cancers among men [1]. This study examined the relationship between individual- and area-level factors and HPV vaccine initiation and completion for boys in the U.S. to guide the development of targeted interventions to improve HPV 


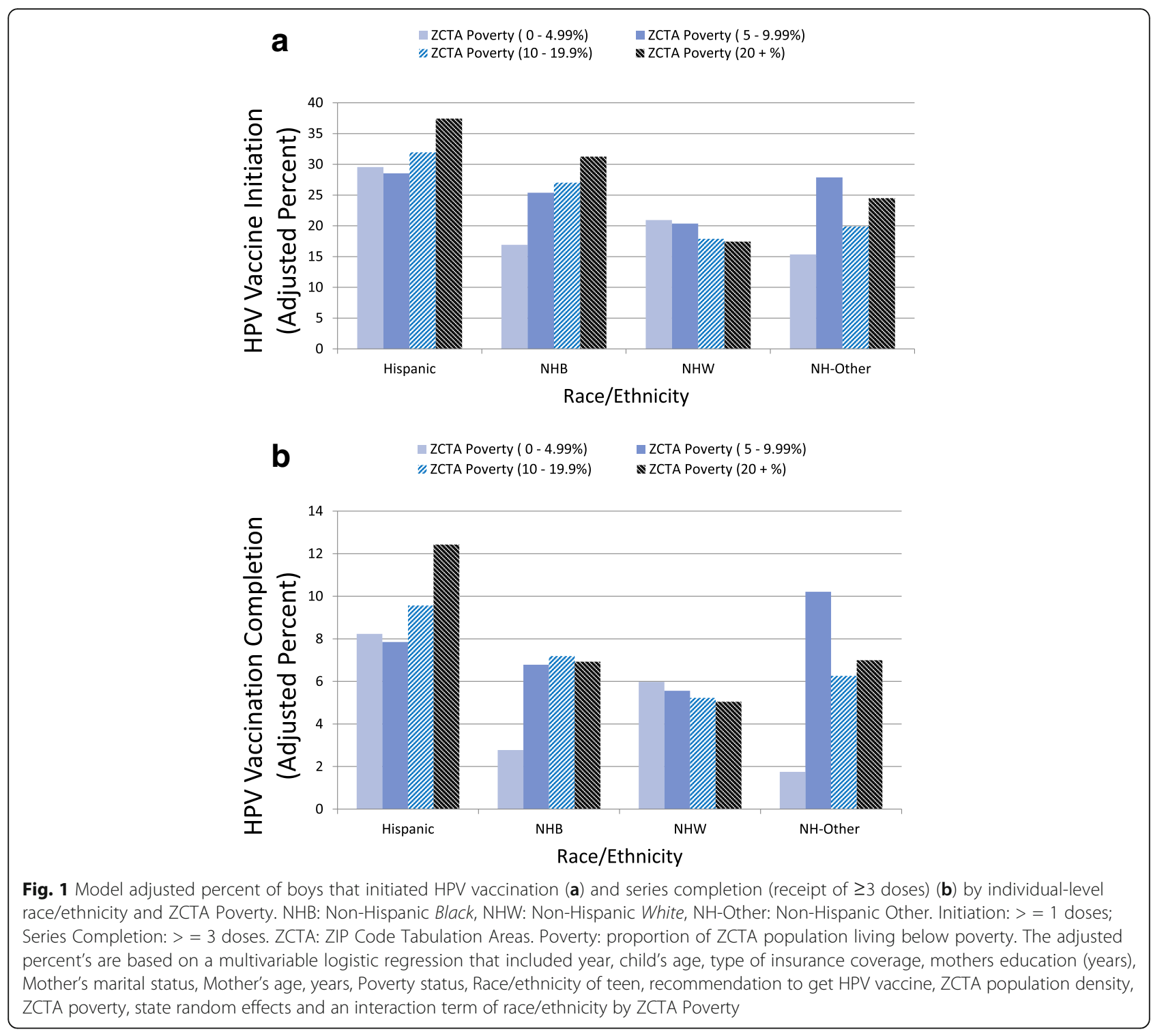

vaccination for this understudied population. Our findings are consistent with existing literature on girls in that the odds of initiation and completion were higher for boys on Medicaid/SCHIP, boys who received a provider recommendation to receive the vaccine, boys from households with incomes below the poverty threshold, and boys who were Hispanic and NHB [40, 57-60]. However, we found that the odds of both HPV vaccine initiation and completion for boys varied by their race/ ethnicity depending on level of poverty in the ZCTA where they lived. We also found that boys living in urban areas had higher odds of both initiation and completion compared to boys living in non-urban areas, and that boys from areas where the majority race/ethnicity group was Hispanic had greater odds of vaccine initiation compared to boys from majority NHW or NHB areas.
Our finding that urban and high-population density areas had higher vaccination coverage compared to rural and suburban areas was generally consistent with prior NIS-Teen studies that used Metropolitan Statistical Areas, which are much larger geographic areas than ZCTAs [61]. These studies examined differences between teens living in urban or metropolitan areas and teens living in rural or non-metropolitan areas, and produced mixed results [62-64]. However, most of these studies used different rural and urban definitions based on large heterogeneous geographic regions (e.g., Metropolitan Statistical Areas). In our study, we used ZCTA, which is a smaller, more defined homogeneous geographic unit. This approach identified populations with higher odds of HPV vaccination among teen boys in urban areas, which could be the result of shorter distances to sources of care, higher density of safety-net 


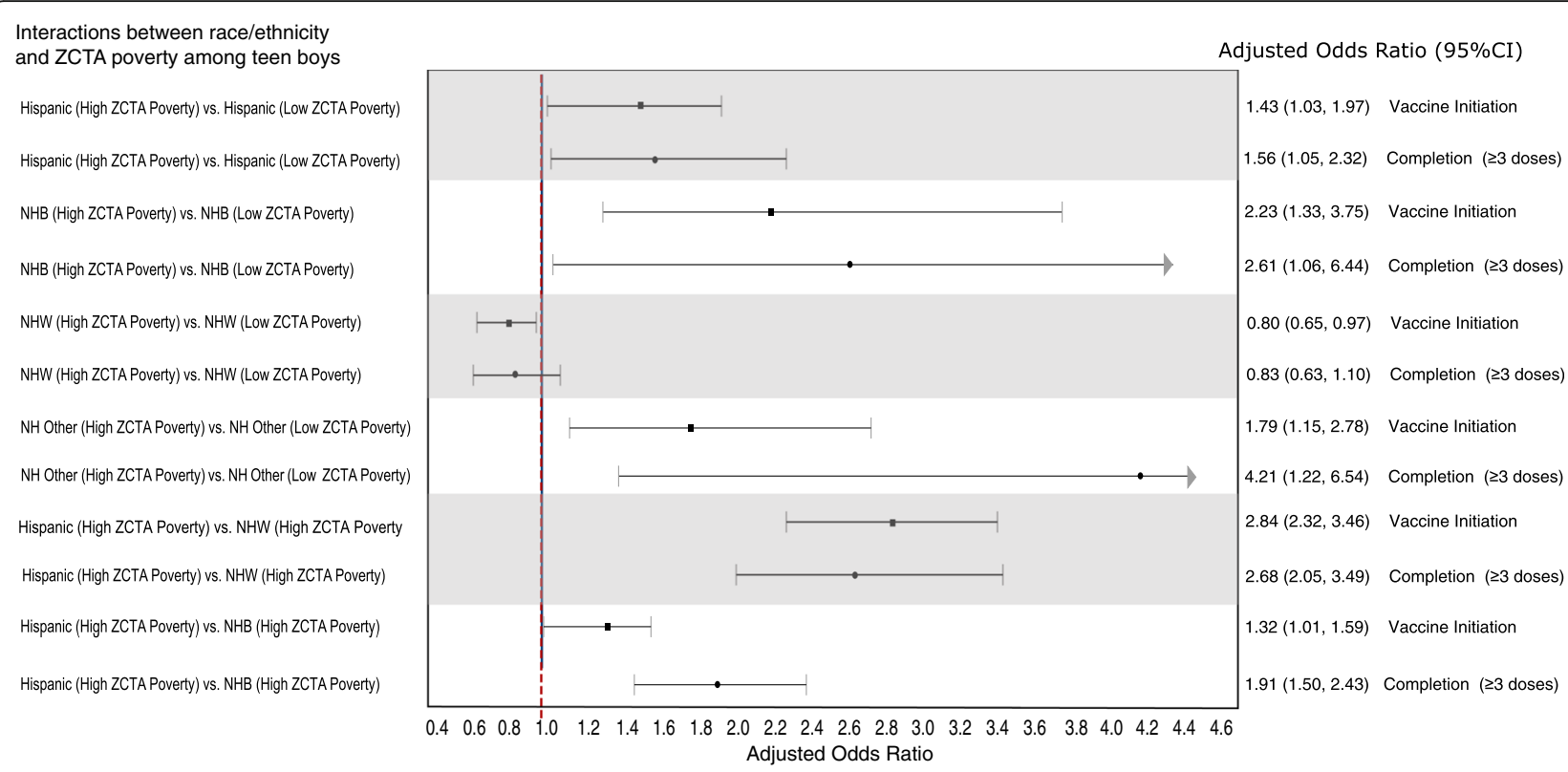

Fig. 2 Adjusted Odds of initiation (Receipt of at Least One Dose) and completion (receipt of $\geq 3$ doses) among male Adolescents Aged 13 to 17 Years and Their Families: National Immunization Survey-Teen, 2012-2013. Results based on statistically significant interactions between Race/ethnicity and ZCTA Poverty for HPV Vaccine Initiation. Note/legend: The adjusted odds ratios are based on a multivariable logistic regression that included year, teen's age in years, type of insurance coverage, mothers education (years), Mother's marital status, Mother's age, survey year, poverty status, race/ethnicity of teen, recommendation to get HPV vaccine, ZCTA population density quartile, ZCTA poverty, state random effects and a interaction term of race/ethnicity by ZCTA poverty

services, and more favorable opinions about HPV vaccination. Additionally, the higher HPV vaccination coverage could also be a result of a greater proportion of providers in urban areas who are more frequently recommending the HPV vaccine than those in rural or suburban areas [65-67]. More research is needed to further delineate the relationship between residence type (e.g. urban vs. suburban vs. rural) and delivery of preventive health services, such as the HPV vaccine.

The higher odds of HPV vaccine initiation for Hispanics and NHB boys from areas with high levels of poverty is likely a reflection of access to public health safety-net services, targeted community-based interventions [68-70], and an undercurrent of cultural factors that help to promote HPV vaccination. The gap in vaccination in less impoverished areas may also be due to few (if any) health services support for HPV vaccination in wealthier areas; the Vaccines for Children program, which is only available to lowincome children; and, targeted preventative health education programs, which have been shown to increase vaccination rates in low-income minority areas [71]. Physicians in low-income areas may also promote the HPV vaccine among those eligible or already enrolled in the Vaccines for Children program and receiving other vaccines during a physician visit (e.g., concomitant vaccination) [72].
The lower prevalence of HPV vaccine initiation among boys in more affluent areas, irrespective of race/ethnicity, is also likely due to less parental support of HPV vaccination as compared to parents from lower-SES groups [73]. It is also possible that both NHW and racial/ethnic minority parents living in affluent areas are more exposed to negative sentiment or vaccination safety concerns and may not advocate vaccinating their sons against HPV [74]. Noting the importance of physician recommendation, it is possible that physicians in wealthier areas either fail to or inconsistently recommend the HPV vaccine more frequently compared to physicians from high-poverty areas [75]. More research is needed to better understand how area-based poverty level impacts delivery of preventive health services for teens.

The overall low rates of HPV vaccine initiation and completion are a concern, especially when compared to the rates of other recommended vaccines for teens. The influenza vaccine, like the HPV vaccine, is recommended but not required by ACIP for 13- to 17-year-olds [76]. For the 2012-2013 influenza season, 45.1\% of Hispanic children aged 13-17 years received the flu vaccine, with similar rates of $44.1 \%$ for NHB and $41.4 \%$ for NHW [77] of the same age. While the flu vaccine rates, like those for HPV vaccine rates, do not meet the goals of the Healthy People 2020 initiative, we do not see flu vaccine rates as low as HPV vaccine rates, and differences 
Table 3 Odds of HPV Vaccine series completion (receipt of $\geq 3$ doses) among of Male Adolescents 13 to 17 Years of age for Individual-level and area-based measures: National Immunization Survey-Teen, 2012-2013

\begin{tabular}{|c|c|c|c|c|}
\hline \multirow[b]{2}{*}{ Characteristics } & \multicolumn{2}{|c|}{ HPV vaccine series completion ${ }^{a}$} & \multirow[b]{2}{*}{$\begin{array}{l}\text { Individual-level + ZCTA } \\
\text { racial composition + ZCTA } \\
\text { population density } \\
\text { (Model 2), AOR }{ }^{\text {a, b }}(95 \% \mathrm{Cl})\end{array}$} & \multirow[b]{2}{*}{$\begin{array}{l}\text { Individual-level + ZCTA } \\
\text { poverty, + ZCTA population } \\
\text { density (Model 3), } \\
\text { AOR }^{\mathrm{a}, \mathrm{b}}(95 \% \mathrm{Cl})\end{array}$} \\
\hline & $\begin{array}{l}\text { Undadjusted }{ }^{\mathrm{b}, \mathrm{c}} \\
\text { OR }(95 \% \mathrm{Cl})\end{array}$ & $\begin{array}{l}\text { Individual-level only, } \\
\text { (Model 1) AOR a, b } \\
(95 \% \text { Cl) }\end{array}$ & & \\
\hline \multicolumn{5}{|l|}{ Individual-level variables } \\
\hline \multicolumn{5}{|l|}{ Teens Age } \\
\hline 13 & Ref & Ref & Ref & Ref \\
\hline 14 & $1.04(0.89,1.21)$ & $1.06(0.91,1.25)$ & $1.08(0.92,1.27)$ & $1.05(0.90,1.24)$ \\
\hline 15 & $1.32(1.14,1.52)^{*}$ & $1.50(1.29,1.75)^{* *}$ & $1.48(1.27,1.73)^{* *}$ & $1.48(1.27,1.73)^{* *}$ \\
\hline 16 & $1.09(0.84,1.27)$ & $1.16(0.99,1.37)$ & $1.16(0.98,1.36)$ & $1.15(0.98,1.35)$ \\
\hline 17 & $1.27(1.10,1.48)^{*}$ & $1.48(1.26,1.74)^{* *}$ & $1.46(1.24,1.71)^{* *}$ & $1.45(1.23,1.70)^{* *}$ \\
\hline \multicolumn{5}{|l|}{ Mother's marital status } \\
\hline Married & Ref & Ref & Ref & Ref \\
\hline Not married & $1.11(1.01,1.22)^{*}$ & $0.93(0.83,1.04)$ & $0.94(0.84,1.05)$ & $0.93(0.83,1.04)$ \\
\hline \multicolumn{5}{|l|}{ Type of insurance coverage insurance } \\
\hline No insurance & $0.65(0.52,0.81)^{*}$ & $0.60(0.47,0.76)^{* *}$ & $0.59(0.46,0.76)^{* *}$ & $0.59(0.46,0.75)^{* *}$ \\
\hline Employer or union & Ref & Ref & Ref & Ref \\
\hline SCHIP or Medicaid & $1.69(1.52,1.87)^{* *}$ & $1.37(1.18,1.59)^{* *}$ & $1.38(1.18,1.60)^{* *}$ & $1.36(1.17,1.58)^{* *}$ \\
\hline IHS, military, other & $1.35(1.16,1.56)^{* *}$ & $1.27(1.08,1.49)^{*}$ & $1.30(1.10,1.53)^{*}$ & $1.25(1.06,1.47)^{*}$ \\
\hline \multicolumn{5}{|l|}{ Mother's education, years } \\
\hline$<12$ years & $1.90(167,2.16)^{* *}$ & $1.73(1.45,2.07)^{* *}$ & $1.77(1.48,2.13)^{* *}$ & $1.69(1.41,2.03)^{* *}$ \\
\hline 12 years & $0.95(0.83,1.07)$ & $0.90(0.77,1.05)$ & $0.91(0.78,1.07)$ & $0.90(0.77,1.05)$ \\
\hline$>12$ years, non-college graduate & $0.88(0.78,1.00)$ & $0.90(0.78,1.04)$ & $0.92(0.79,1.05)$ & $0.90(0.78,1.04)$ \\
\hline College graduate & Ref & Ref & Ref & Ref \\
\hline \multicolumn{5}{|l|}{ Poverty status } \\
\hline Above poverty, $>\$ 75,000$ & Ref & Ref & Ref & Ref \\
\hline Above poverty, $\leq \$ 75,000$ & $1.08(0.96,1.21)$ & $0.99(0.86,1.14)$ & $0.99(0.85,1.14)$ & $0.10(0.86,1.15)$ \\
\hline Below poverty & $1.58(1.40,1.79)^{* *}$ & $1.11(0.93,1.34)$ & $1.13(0.93,1.36)$ & $1.11(0.92,1.34)$ \\
\hline Unknown poverty status & $1.21(0.96,1.53)$ & $0.91(0.70,1.19)$ & $0.90(0.69,1.18)$ & $0.90(0.69,1.17)$ \\
\hline \multicolumn{5}{|l|}{ Race/ethnicity of teen } \\
\hline Hispanic & $2.19(1.95,2.46)^{* *}$ & $1.95(1.70,2.24)^{* *}$ & $1.89(1.62,2.19)^{* *}$ & $1.86(1.61,2.15)^{* *}$ \\
\hline Non-Hispanic White & Ref & Ref & Ref & Ref \\
\hline Non-Hispanic Black & $1.44(1.24,1.66)^{* *}$ & $1.26(1.08,1.48)^{*}$ & $1.21(1.01,1.44)^{*}$ & $1.19(1.01,1.40)^{*}$ \\
\hline Non-Hispanic other and multiple race & $1.24(1.03,1.48)^{*}$ & $1.22(1.01,1.47)^{*}$ & $1.24(1.03,1.51)^{*}$ & $1.20(0.10,1.45)$ \\
\hline \multicolumn{5}{|c|}{ Received provider recommendation to get HPV vaccine } \\
\hline Yes & $6.59(5.89,7.36)^{* *}$ & $6.53(5.83,7.33)^{* *}$ & $6.60(5.89,7.42)^{* *}$ & $6.53(5.82,7.33)^{* *}$ \\
\hline No & Ref & Ref & Ref & Ref \\
\hline Don't know & $2.20(1.78,2.71)^{* *}$ & $2.19(1.77,2.70)^{* *}$ & $2.20(1.77,2.73)^{* *}$ & $2.19(1.78,2.71)^{* *}$ \\
\hline \multicolumn{5}{|l|}{ ZCTA Area-based measures } \\
\hline \multicolumn{5}{|l|}{ Racial composition ( $50 \%$ + of that group) } \\
\hline Hispanic & $1.88(1.62,2.19)$ & - & $0.98(0.81,1.18)$ & - \\
\hline Mixed & $1.58(1.38,1.81)$ & - & $1.16(0.99,1.36)$ & - \\
\hline Non-Hispanic Black & $1.23(1.00,1.51)$ & - & $0.99(0.78,1.23)$ & - \\
\hline Non-Hispanic White & Ref & - & Ref & Ref \\
\hline
\end{tabular}


Table 3 Odds of HPV Vaccine series completion (receipt of $\geq 3$ doses) among of Male Adolescents 13 to 17 Years of age for Individual-level and area-based measures: National Immunization Survey-Teen, 2012-2013 (Continued)

\begin{tabular}{|c|c|c|c|c|}
\hline Non-Hispanic other & $0.60(0.32,1.14)$ & - & $0.27(0.14,0.54)^{*}$ & - \\
\hline \multicolumn{5}{|l|}{ Poverty (\% below poverty) ${ }^{d}$} \\
\hline 0-4.99\%, least impoverished & Ref & - & - & Ref \\
\hline $5-9.9 \%$ & $1.08(0.92,1.28)$ & - & - & $1.09(0.92,1.30)$ \\
\hline $10-19.9 \%$ & $1.08(0.92,1.27)$ & - & - & $1.05(0.88,1.25)$ \\
\hline $20+\%$, poorest & $1.58(1.34,1.86)^{* *}$ & - & - & $1.22(1.01,1.48)^{*}$ \\
\hline \multicolumn{5}{|l|}{ Population density quartiles (people per sqr mile) } \\
\hline 1, lowest density) 1-20 per sq. mile & Ref & - & Ref & Ref \\
\hline 2. 21-71 persons per sq. mile & $0.71(1.00,0.50)$ & - & $0.60(0.42,0.86)^{*}$ & $0.61(0.42,0.86)^{*}$ \\
\hline 3. $72-651$ persons per sq. mile & $1.19(0.88,1.62)$ & - & $1.01(0.73,1.39)$ & $1.02(0.74,1.40)$ \\
\hline 4. highest density $>651$ person per sq. mile & $1.34(0.99,1.82)$ & & $0.95(0.69,1.30)$ & $0.95(0.69,1.30)$ \\
\hline Q4 vs Q2 & $1.90(1.55,2.32)^{* *}$ & & $1.57(1.27,1.94)^{* *}$ & $1.57(1.27,1.94)^{* *}$ \\
\hline Q4 vs Q3 & $1.13(1.01,1.26)^{*}$ & & $0.94(083,1.06)$ & $0.93(0.83,1.05)$ \\
\hline \multicolumn{5}{|l|}{ Residence type } \\
\hline 1. Isolated Small Rural T & Ref & & - & - \\
\hline 2. Small Rural Town & $1.05(0.61,1.80)$ & & - & - \\
\hline 3. Large Rural Town & $1.25(0.77,2.01$ & & - & - \\
\hline 4. Urban focused & $1.92(1.26,2.92)^{*}$ & & - & - \\
\hline
\end{tabular}

Note CI confidence interval, HPV human papillomavirus, IHS Indian Health, SCHIP State Children's Health Insurance Program, Ref Reference, AOR adjusted odds ratio

${ }^{*} P<.05 ; * * P<.0001$

${ }^{a}$ completion $=>=3$ doses

b Unadjusted odds and models 1-3 were weighted based on sampling weight and also included state random effects. The sample size was $n=19,188$

' Multivariable models 1-3 include all of the variables without dashes $(-)$ and also include the variables, marital status, mother's age, survey year, and teen's current age in years and state random effects

${ }^{d}$ Poverty status was based on the US Census poverty thresholds for 2012 and 2013

between racial/ethnic groups are not as large as with the HPV vaccine [78]. This indicates that even among other recommended vaccines, HPV vaccine is a unique case and may require different interventions to achieve public health goals.

Indeed, our study suggests that one approach would be to conduct community health assessments and environmental scans in areas that are not only predominantly Hispanic but also to target teen boys, their parents, and their health care providers in high-poverty areas to gather evidenced-based practices to improve dissemination and intervention of HPV vaccination initiatives in other areas. A promising area is in an examination of the effects of acculturation. Studies have suggested that lower levels of acculturation tend to be more common among poor Hispanics $[79,80]$, and that Hispanics with lower acculturation are more supportive of vaccination, which may account for the differences seen among Hispanics from low-income areas compared to Hispanics from wealthier areas [81, 82]. Targeted safety-net services and interventions have been traditionally focused on low-income areas, but our study suggests that further geographic and racial/ethnic factors alter adoption of vaccines, which requires additional research.
Higher HPV vaccination prevalence among Hispanics is one of the more successful initiatives in the fight against HPV-related cancers in both males and females. Hispanic men are more likely to be infected with multiple types of HPV, which are associated with longer HPV infections and more precancerous lesions [57]. Hispanic males also have higher rates of penile cancer than non-Hispanic males have. Thus, higher HPV vaccine rates could help prevent these cancers in the Hispanic male population.

Our study has several limitations. First, by using crosssectional data, there is potential of misclassification of series completion. Bias is possible if boys were classified as having received only one or two doses (i.e., mid-series at time of survey), but later completed the three-dose series within the recommended duration. Second, the parents' role in the survey is subject to recall bias because some parents may have incorrectly recalled whether they had ever received a provider recommendation for the vaccine. Finally, ZCTA was the smallest unit available for the NIS-Teen survey, which has been shown to be more heterogeneous than smaller units such as census tracts [83]. Using different geographic units may yield different results (e.g., the modifiable unit problem). 


\section{Conclusion}

This study found that HPV vaccination coverage for boys varied by their race/ethnicity depending on the level of poverty in their ZIP Code Tabulation Area. Racial/ethnic minorities from areas with high levels of poverty had higher odds of both HPV vaccination initiation and completion compared to those from low-poverty areas. We also found that boys from areas where the majority race/ethnicity group was Hispanic had greater odds of vaccine initiation compared to boys from majority NHW or NHB areas. Higher HPV vaccination coverage in areas with high poverty may be attributable to targeted interventions by the Vaccines for Children program, which provides free recommended vaccines to uninsured and under-insured children. Given the low HPV vaccination rates in the U.S., these results provide important evidence to inform public health interventions to increase HPV vaccination.

\section{Abbreviations \\ ACIP: Advisory Committee on Immunization Practices; AOR: Adjusted odds ratio; CDC: Centers for Disease Control and Prevention (CDC); HPV: Human papilloma virus; NHB: Non-Hispanic black; NHW: Non-Hispanic white; NIS-T: National immunization survey teen; SCHIP: State Children's Health Insurance Program; VFC: Vaccine foe children; ZCTA: ZIP Code tabulation area}

\section{Acknowledgements}

Not Applicable.

\section{Funding}

This study was supported in part by NIH/NCI Grant \# 1R03CA202566-01, the Huntsman Cancer Foundation, and the Beaumont Foundation. The findings and conclusions in this report are those of the authors and do not necessarily represent the official positions of the National Institutes of Health, Huntsman Cancer Institute Foundation or Fox Chase Cancer Center.

\section{Availability of data and materials}

The non-geographic data used for this study is available publicly as part of the NCHS National Immunization Survey data. https://www.cdc.gov/nchs/nis/ data_files_teen.htm. The census data used for this study is also available publicly http://www.census.gov/programs-surveys/acs/. The file containing the geographic (ZIP Code) based census data merged to the NIS-T survey data is restricted use data and researchers would have to submit a proposal to NCHS to access the merged the datasets on site at NCHS Research Data Center.

\section{Disclaimer}

The findings and conclusions in this paper are those of the author(s) and do not necessarily represent the views of the Research Data Center, the National Center for Health Statistics, or the Centers for Disease Control and Prevention.

\section{Authors' contributions}

$\mathrm{KH}, \mathrm{EW}$, DK conceived the study and acquired the data. AA and $\mathrm{KH}$ drafted the manuscript. All authors made substantial contributions to the study the design, the interpretation of results and revision of the manuscript. All the authors read, edited and approved the final manuscript.

\section{Ethics approval and consent to participate}

Data collection for National Immunization Survey Teen (NIS-T) was approved by the United States National Center for Health Statistics (NCHS) Research Ethics Review Board. Analysis of de-identified data from the survey is exempt from the federal regulations for the protection of human research participants. Analysis of restricted data through the NCHS Research Data Center is also approved by the NCHS ERB. The Institutional Review Board at Temple University determined this study was exempt from review.
Consent for publication

Not applicable.

\section{Competing interests}

The authors declare that they have no competing interests.

\section{Publisher's Note}

Springer Nature remains neutral with regard to jurisdictional claims in published maps and institutional affiliations.

\section{Author details}

${ }^{1}$ Department of Geography, Temple University, 115 W. Polett Walk, 308 Gladfelter Hall, Philadelphia, PA 19122, USA. ${ }^{2}$ Fox Chase Cancer Center, Cancer Prevention and Control Program, 333 Cottman Avenue, Philadelphia, PA 19111, USA. ${ }^{3}$ Temple University, Lewis Katz School of Medicine, 3500 North Broad Street, Philadelphia, PA 19140, USA. ${ }^{4}$ Department of Epidemiology, Division of Cancer Epidemiology, New Jersey State Cancer Registry, Rutgers University, Rutgers School of Public Health, 683 Hoes Lane West, Piscataway, NJ 08854, USA. ${ }^{5}$ Cancer Institute of New Jersey, Rutgers University, Cancer Prevention and Control Program, 195 Little Albany Street, New Brunswick, NJ 08903, USA. 'Huntsman Cancer Institute, University of Utah, Cancer Control and Population Sciences, 2000 Circle of Hope, Salt Lake City, UT 84112, USA. 'University of Utah College of Nursing, 10 South 2000 East, Salt Lake City, UT 84112, USA.

Received: 22 December 2016 Accepted: 5 July 2017

Published online: 14 July 2017

\section{References}

1. Viens $L$, Henley SJ, Watson M, Markowitz LE, Thomas CC, Thompson TD, Razzaghi H, Saraiya M. Human Papillomavirus-associated cancers - United States, 2008-2012. MMWR Morb Mortal Wkly Rep. 2016;65(26):661-6.

2. Dunne EF, Unger ER, Sternberg M, McQuillan G, Swan DC, Patel SS, Markowitz LE. Prevalence of HPV infection among females in the United States. JAMA. 2007;297(8):813-9.

3. Chaturvedi AK, Engels EA, Pfeiffer RM, Hernandez BY, Xiao W, Kim E, Jiang B, Goodman MT, Sibug-Saber M, Cozen W, et al. Human papillomavirus and rising oropharyngeal cancer incidence in the United States. J Clin Oncol. 2011;29(32):4294-301.

4. Ryerson AB, Eheman CR, Altekruse SF, Ward JW, Jemal A, Sherman RL, Henley SJ, Holtzman D, Lake A, Noone AM, et al. Annual report to the nation on the status of cancer, 1975-2012, featuring the increasing incidence of liver cancer. Cancer. 2016;122(9):1312-37.

5. Jemal A, Simard EP, Dorell C, Noone AM, Markowitz LE, Kohler B, Eheman C, Saraiya M, Bandi P, Saslow D, et al. Annual report to the nation on the status of cancer, 1975-2009, featuring the burden and trends in human papillomavirus(HPV)-associated cancers and HPV vaccination coverage levels. J Natl Cancer Inst. 2013;105(3):175-201.

6. Boscoe FP, Johnson CJ, Sherman RL, Stinchcomb DG, Lin G, Henry KA. The relationship between area poverty rate and site-specific cancer incidence in the United States. Cancer. 2014;120(14):2191-8.

7. Kuehn BM. CDC panel backs routine HPV vaccination. JAMA. 2006;296(6):640-1.

8. Markowitz LE, Dunne EF, Saraiya M, Chesson HW, Curtis CR, Gee J, Bocchini JA Jr, Unger ER. Human papillomavirus vaccination: recommendations of the advisory committee on immunization practices (ACIP). MMWR Recomm Rep. 2014;63(RR-05):1-30.

9. CDC. Human Papillomavirus. In: Hamborsky J, Kroger A, Wolfe S, editors. Centers for Disease Control and Prevention epidemiology and prevention of vaccine-preventable diseases. 13th ed. Washington D.C: Public Health Foundation; 2015.

10. "HPV Vaccine Information for Clinicians - Fact Sheet." Centers for Disease Control and Prevention. http://www.cdc.gov/std/hpv/stdfact-hpv-vaccinehcp.htm. Accessed 07 July 2016.

11. Meites E, Kempe A, Markowitz LE. Use of a 2-dose schedule for human Papillomavirus vaccination - updated recommendations of the advisory committee on immunization practices. MMWR Morb Mortal Wkly Rep. 2016;65(49):1405-8.

12. National Cancer Institute. Accelerating HPV vaccine uptake: urgency for action to prevent cancer. A report to the president of the United States from the President's cancer panel. Bethesda: National Cancer Institute; 2014. 
13. CDC. Human papillomavirus-associated cancers - United States, 2004-2008. MMWR Morb Mortal Wkly Rep. 2012;61:258-61.

14. Kessels SJ, Marshall HS, Watson M, Braunack-Mayer AJ, Reuzel R, Tooher RL. Factors associated with HPV vaccine uptake in teenage girls: a systematic review. Vaccine. 2012;30(24):3546-56.

15. Holman DM, Benard V, Roland KB, Watson M, Liddon N, Stokley S. Barriers to human papillomavirus vaccination among US adolescents: a systematic review of the literature. JAMA Pediatr. 2014;168(1):76-82.

16. Warner ET, Gomez SL. Impact of neighborhood racial composition and metropolitan residential segregation on disparities in breast cancer stage at diagnosis and survival between black and white women in California. J Community Health. 2010;35(4):398-408.

17. Merkin SS, Stevenson L, Powe N. Geographic socioeconomic status, race, and advanced-stage breast cancer in new York City. Am J Public Health. 2002;92(1):64-70.

18. Shariff-Marco S, Yang J, John EM, Sangaramoorthy M, Hertz A, Koo J, Nelson DO, Schupp CW, Shema SJ, Cockburn M, et al. Impact of neighborhood and individual socioeconomic status on survival after breast cancer varies by race/ethnicity: the neighborhood and breast cancer study. Cancer Epidemiol Biomarkers Prev. 2014;23(5):793-811.

19. Lantz PM, Mujahid M, Schwartz K, Janz NK, Fagerlin A, Salem B, Liu L, Deapen D, Katz SJ. The influence of race, ethnicity, and individual socioeconomic factors on breast cancer stage at diagnosis. Am J Public Health. 2006;96(12):2173-8.

20. Henry KA, McDonald K, Sherman R, Kinney AY, Stroup AM. Association between individual and geographic factors and nonadherence to mammography screening guidelines. J Women's Health (Larchmt). 2014;23(8):664-74.

21. Walker AT, Smith PJ, Kolasa M, Centers for Disease C, Prevention. Reduction of racial/ethnic disparities in vaccination coverage, 1995-2011. MMWR Surveill Summ. 2014;63(Suppl 1):7-12.

22. Malo TL, Hassani D, Staras SA, Shenkman EA, Giuliano AR, Vadaparampil ST. Do Florida Medicaid providers' barriers to HPV vaccination vary based on VFC program participation? Matern Child Health J. 2013;17(4):609-15.

23. Arcury TA, Gesler WM, Preisser JS, Sherman J, Spencer J, Perin J. The effects of geography and spatial behavior on health care utilization among the residents of a rural region. Health Serv Res. 2005;40(1):135-55.

24. Field K. Measuring the need for primary health care: an index of relative disadvantage. Appl Geogr. 2000;20(4):305-32.

25. Freeman $\mathrm{HE}$, Corey $\mathrm{CR}$. Insurance status and access to health services among poor persons. Health Serv Res. 1993;28(5):531-41.

26. Cui Y, Baldwin SB, Wiley DJ, Fielding JE. Human papillomavirus vaccine among adult women: disparities in awareness and acceptance. Am J Prev Med. 2010;39(6):559-63.

27. Jain N, Singleton JA, Montgomery M, Skalland B. Determining accurate vaccination coverage rates for adolescents: the National Immunization Survey-Teen 2006. Public Health Rep. 2009;124(5):642-51.

28. Marlow LA, Wardle J, Forster AS, Waller J. Ethnic differences in human papillomavirus awareness and vaccine acceptability. J Epidemiol Community Health. 2009;63(12):1010-5.

29. Glenn BA, Tsui J, Singhal R, Sanchez L, Nonzee NJ, Chang LC, Taylor VM, Bastani R. Factors associated with HPV awareness among mothers of low-income ethnic minority adolescent girls in Los Angeles. Vaccine. 2015;33(2):289-93.

30. Buster KJ, You Z, Fouad M, Elmets C. Skin cancer risk perceptions: a comparison across ethnicity, age, education, gender, and income. J Am Acad Dermatol. 2012;66(5):771-9.

31. Johnson S, McDonald JT, Corsten M. Oral cancer screening and socioeconomic status. J Otolaryngol Head Neck Surg. 2012;41(2):102-7.

32. Katz SJ, Hofer TP. Socioeconomic disparities in preventive care persist despite universal coverage. Breast and cervical cancer screening in Ontario and the United States. JAMA. 1994;272(7):530-4.

33. Phillips KA, Liang SY, Ladabaum U, Haas J, Kerlikowske K, Lieberman D, Hiatt R, Nagamine M, Van Bebber SL. Trends in colonoscopy for colorectal cancer screening. Med Care. 2007:45(2):160-7.

34. Pruitt SL, Shim MJ, Mullen PD, Vernon SW, Amick BC 3rd. Association of area socioeconomic status and breast, cervical, and colorectal cancer screening: a systematic review. Cancer Epidemiol Biomarkers Prev. 2009;18(10):2579-99.

35. Henry KA, Stroup AM, Warner EL, Kepka D. Geographic factors and human Papillomavirus (HPV) vaccination initiation among adolescent girls in the United States. Cancer Epidemiol Biomarkers Prev. 2016;25(2):309-17.
36. Centers for Disease Control and Prevention. Teen Vaccination Coverage. A User's Guide for the 2013 Public-Use Data File National Immunization Survey (NIS) - Teen, ftp://ftp.cdc.gov/pub/Health_Statistics/NCHS/ Dataset_Documentation/NIS/NISTEENPUF13_DUG.pdf; 2014. Accessed 7 May 2017.

37. Henry K, Warner E, Ding Q, Kepka D. The role of geographic factors in human Papillomavirus (HPV) vaccine uptake among adolescent girls in the United States. Cancer Epidemiol Biomark Prev. 2015;24:758.

38. Moss JL, Gilkey MB, Reiter PL, Brewer NT. Trends in HPV vaccine initiation among adolescent females in North Carolina, 2008-2010. Cancer Epidemiol Biomarkers Prev. 2012;21(11):1913-22

39. Reiter PL, Brewer NT, Gilkey MB, Katz ML, Paskett ED, Smith JS. Early adoption of the human papillomavirus vaccine among Hispanic adolescent males in the United States. Cancer. 2014;120(20):3200-7.

40. Reiter PL, Gilkey MB, Brewer NT. HPV vaccination among adolescent males: results from the National Immunization Survey-Teen. Vaccine. 2013;31(26):2816-21.

41. Ylitalo KR, Lee H, Mehta NK. Health care provider recommendation, human papillomavirus vaccination, and race/ethnicity in the US National Immunization Survey. Am J Public Health. 2013;103(1):164-9.

42. U.S. Census Bureau. Poverty Thresholds by Size of Family and Number of Related Children Under 18 Years, 2011-2012. In. U.S. Census Bureau Website: https://www.census.gov/topics/income-poverty/poverty/data/tables.html U.S. Census Bureau; 2013. Accessed 07 July 2016.

43. US Census Bureau. ZIP Code Tabulation Areas (ZCTAs),. In. US Census Bureau Website, https://www.census.gov/geo/reference/zctas.html: US Census Bureau; 2011. Accessed 7 July 2016.

44. Krieger N, Chen JT, Waterman PD, Rehkopf DH, Subramanian SV. Race/ ethnicity, gender, and monitoring socioeconomic gradients in health: a comparison of area-based socioeconomic measures-the public health disparities geocoding project. Am J Public Health. 2003;93(10):1655-71.

45. Li K, Wen M, Henry KA. Residential racial composition and black-white obesity risks: differential effects of neighborhood social and built environment. Int J Environ Res Public Health. 2014;11(1):626-42.

46. Anderson AE, Henry KA, Samadder NJ, Merrill RM, Kinney AY. Rural vs urban residence affects risk-appropriate colorectal cancer screening. Clin Gastroenterol Hepatol. 2013;11(5):526-33.

47. Pruitt SL, Schootman M. Geographic disparity, area poverty, and human papillomavirus vaccination. Am J Prev Med. 2010;38(5):525-33.

48. Tsui J, Singhal R, Rodriguez HP, Gee GC, Glenn BA, Bastani R. Proximity to safety-net clinics and HPV vaccine uptake among low-income, ethnic minority girls. Vaccine. 2013;31(16):2028-34.

49. White K, Borrell LN. Racial/ethnic residential segregation: framing the context of health risk and health disparities. Health Place. 2011;17(2):438-48.

50. Massey DS, Denton NA. The dimensions of residential segregation. Soc Forces. 1988;67:281-315.

51. Russell E, Kramer MR, Cooper HL, Thompson WW, Arriola KR. Residential racial composition, spatial access to care, and breast cancer mortality among women in Georgia. J Urban Health. 2011;88(6):1117-29.

52. Russell EF, Kramer MR, Cooper HL, Gabram-Mendola S, Senior-Crosby D, Jacob Arriola KR. Metropolitan area racial residential segregation, neighborhood racial composition, and breast cancer mortality. Cancer Causes Control. 2012;23(9):1519-27.

53. Gomez SL, Glaser SL, McClure LA, Shema SJ, Kealey M, Keegan TH, Satariano WA. The California neighborhoods data system: a new resource for examining the impact of neighborhood characteristics on cancer incidence and outcomes in populations. Cancer Causes Control. 2011;22(4):631-47.

54. Rural Health Research Center. Rural-Urban Commuting Areas (RUCAs). University of Washington, Retrieved 3 March 2010 from University of Washington: http://depts.washington.edu/uwruca/ruca-rural.php . 2007. Accessed 7 July 2016.

55. SAS Institute Inc., Cary, NC, USA

56. SAS Institute Inc. The GLIMMIX procedure. Cary: SAS Institute; 2005.

57. Nielson CM, Harris RB, Flores R, Abrahamsen M, Papenfuss MR, Dunne EF, Markowitz LE, Giuliano AR. Multiple-type human papillomavirus infection in male anogenital sites: prevalence and associated factors. Cancer Epidemiol Biomarkers Prev. 2009:18(4):1077-83.

58. Laz TH, Rahman M, Berenson AB. Human papillomavirus vaccine uptake among 9-17 year old males in the United States: the National Health Interview Survey, 2010. Hum Vaccin Immunother. 2013;9(4):874-8. 
59. Gilkey MB, Moss JL, McRee AL, Brewer NT. Do correlates of HPV vaccine initiation differ between adolescent boys and girls? Vaccine. 2012;30(41):5928-34.

60. Kepka D, Ding Q, Hawkins AJ, Warner EL, Boucher KM. Factors associated with early adoption of the HPV vaccine in US male adolescents include Hispanic ethnicity and receipt of other vaccines. Prev Med Rep. 2016;4:98-102.

61. Centers for Disease Control and Prevention. 2012, 2013 NIS-Teen Vaccination Coverage Table Data, Vaccination Coverage Levels by Urbanicity. https://www.cdc.gov/vaccines/imz-managers/coverage/nis/teen/ index.html; Accessed 7 July 2016

62. Monnat SM, Rhubart DC, Wallington SF. Differences in human Papillomavirus vaccination among adolescent girls in metropolitan versus non-metropolitan areas: considering the moderating roles of maternal socioeconomic status and health care access. Matern Child Health J. 2016:20(2):315-25.

63. Finney Rutten $L$, Wilson PM, Jacobson DJ, Agunwamba AA, Radecki Breitkopf C, Jacobson RM, St Sauver JL. A population-based study of Sociodemographic and geographic variation in HPV vaccination. Cancer Epidemiol Biomarkers Prev. 2017;26(4):533-40.

64. Reiter PL, Cates JR, McRee AL, Gottlieb SL, Shafer A, Smith JS, Brewer NT. Statewide HPV vaccine initiation among adolescent females in North Carolina. Sex Transm Dis. 2010;37(9):549-56.

65. Allison MA, Dunne EF, Markowitz LE, O'Leary ST, Crane LA, Hurley LP Stokley S, Babbel Cl, Brtnikova M, Beaty BL, et al. HPV vaccination of boys in primary care practices. Acad Pediatr. 2013;13(5):466-74.

66. Kulczycki A, Qu H, Shewchuk R. Primary care Physicians' adherence to guidelines and their likelihood to prescribe the human Papillomavirus vaccine for 11- and 12-year-old girls. Womens Health Issues. 2016;26(1):34-9.

67. Krieger JL, Katz ML, Kam JA, Roberto A. Appalachian and non-Appalachian pediatricians' encouragement of the human papillomavirus vaccine: implications for health disparities. Womens Health Issues. 2012;22(1):e19-26.

68. Perkins RB, Zisblatt L, Legler A, Trucks E, Hanchate A, Gorin SS. Effectiveness of a provider-focused intervention to improve HPV vaccination rates in boys and girls. Vaccine. 2015;33(9):1223-9.

69. CPSTF: Community Preventive Services Task Force. The guide to community preventive services. Increasing appropriate vaccination [Internet]. Atlanta (GA): the Task Force. Available from: http://www.thecommunityguide.org/ vaccines/index.html. Accessed 7 July 2016

70. Berenson AB, Rahman M, Hirth JM, Rupp RE, Sarpong KO. A brief educational intervention increases providers' human papillomavirus vaccine knowledge. Hum Vaccin Immunother. 2015;11(6):1331-6.

71. El-Mohandes AA, Katz KS, El-Khorazaty MN, McNeely-Johnson D, Sharps PW, Jarrett MH, Rose A, White DM, Young M, Grylack L, et al. The effect of a parenting education program on the use of preventive pediatric health care services among low-income, minority mothers: a randomized, controlled study. Pediatrics. 2003;111(6 Pt 1):1324-32.

72. Moss JL, Reiter PL, Brewer NT. Concomitant adolescent vaccination in the $U$. S., 2007-2012. Am J Prev Med. 2016;51(5):693-705.

73. Reich JA. Neoliberal mothering and vaccine refusal: imagined gated communities and the privilege of choice. Gend Soc. 2014;28(5):679-704.

74. Darden PM, Thompson DM, Roberts JR, Hale JJ, Pope C, Naifeh M, Jacobson RM. Reasons for not vaccinating adolescents: National Immunization Survey of teens, 2008-2010. Pediatrics. 2013;131(4):645-51.

75. Gilkey MB, Malo TL, Shah PD, Hall ME, Brewer NT. Quality of physician communication about human papillomavirus vaccine: findings from a national survey. Cancer Epidemiol Biomarkers Prev. 2015;24(11):1673-9.

76. CDC. Influenza. In: Hamborsky J, Kroger A, Wolfe S, editors. Centers for Disease Control and Prevention epidemiology and prevention of vaccine-preventable diseases. 13th ed. Washington D.C: Public Health Foundation; 2015.

77. "Flu Vaccination Coverage, United States, 2012-13 Influenza Season." Centers for Disease Control and Prevention. 2013. http://www.cdc.gov/ flu/fluvaxview/coverage-1213estimates.htm. Accessed 06 July 2016.

78. U.S. Department of Health and Human Services. Healthy people 2020: immunization and infectious diseases objectives. [internet]. Washington (DC): DHHS; [updated 2013 Apr 24; cited 2016 Jul 1]. Available from: http:// www.healthypeople.gov/2020/topicsobjectives2020/objectiveslist. aspx?topicld=23 ; 2013. Accessed 7 July 2016.

79. Fitzgerald N, Himmelgreen D, Damio G, Segura-Perez S, Peng YK, PerezEscamilla R. Acculturation, socioeconomic status, obesity and lifestyle factors among low-income Puerto Rican women in Connecticut, U.S., 1998-1999. Rev Panam Salud Publica. 19(5):306-13.
80. Ayala GX, Baquero B, Klinger S. A systematic review of the relationship between acculturation and diet among Latinos in the United States: implications for future research. J Am Diet Assoc. 2008;108(8):1330-44.

81. Anderson LM, Wood DL, Sherbourne CD. Maternal acculturation and childhood immunization levels among children in Latino families in Los Angeles. Am J Public Health. 1997:87(12):2018-21.

82. Crawford ND, Blaney S, Amesty S, Rivera AV, Turner AK, Ompad DC, Fuller CM. Individual- and neighborhood-level characteristics associated with support of in-pharmacy vaccination among ESAP-registered pharmacies: pharmacists' role in reducing racial/ethnic disparities in influenza vaccinations in new York City. J Urban Health. 2011;88(1):176-85.

83. Krieger N, Waterman P, Chen JT, Soobader MJ, Subramanian SV, Carson R. Zip code caveat: bias due to spatiotemporal mismatches between zip codes and US census-defined geographic areas-the public health disparities Geocoding project. Am J Public Health. 2002;92(7):1100-2.

\section{Submit your next manuscript to BioMed Central and we will help you at every step:}

- We accept pre-submission inquiries

- Our selector tool helps you to find the most relevant journal

- We provide round the clock customer support

- Convenient online submission

- Thorough peer review

- Inclusion in PubMed and all major indexing services

- Maximum visibility for your research

Submit your manuscript at www.biomedcentral.com/submit
) Biomed Central 